\title{
Stéroïdes anabolisants : usage thérapeutique ou usage détourné ?
}

\section{Anabolic steroids: therapeutic use or misuse?}

\section{Marie BRESSON, Vincent CIRIMELE*, Marion VILLAIN, Pascal KINTZ}

Laboratoire ChemTox, 3, rue Grüninger - 67400 Illkirch - France

*Auteur à qui adresser la correspondance : Vincent CIRIMELE, Laboratoire ChemTox, 3 , rue Grüninger 67400 Illkirch - France - Tél : +33 390400540 - Fax : +33 390400541 - E-mail : vcirimele@labochemtox.com

\section{RÉSUMÉ}

Les stéroïdes anabolisants androgènes, qu'ils soient exogènes ou endogènes; comptent 41 substances réglementées par l'annexe modifiée selon l'arrêté du 25 mars 2005, mais incluent aussi les molécules dont la structure chimique et/ou les effets pharmacologiques sont similaires.

A ce jour, plus de 40 principes actifs sont recensés par le guide Biam représentant ainsi plusieurs dizaines de spécialités pharmaceutiques essentiellement commercialisées aux USA, en Allemagne, en Italie, aux Pays-Bas, en Suisse, en Angleterre et en Belgique. En France, de nombreuses spécialités contenant ces principes actifs ont été retirées $d u$ marché, mais cette carence est largement comblée par l'explosion de sites Internet proposant ces produits.

Les stéroüdes anabolisants sont essentiellement utilisés par les sportifs pour accroître la masse musculaire et la matrice osseuse, mais aussi pour augmenter l'agressivité et réduire le temps de récupération entre les efforts. En parallèle, leur utilisation dans le but d'augmenter les performances comporte aussi de nombreux effets secondaires : irritabilité de l'individu, atrophies des organes génitaux (testicules, ovaires), développement de cancers hormono-dépendants (cancer du sein, des testicules ou de la prostate), etc.

Comme pour les médicaments ou les stupéfiants, l'intérêt de doser ces substances de la performance dans les échantillons biologiques est multiple et s'inscrit aussi bien dans le cadre clinique, sportif que médicolégal. Par ailleurs, la loi relative à la protection de la santé des sportifs et à la lutte contre le dopage (Loi Buffet), mentionne que le contrôle des agissements interdits peut s'effectuer à partir de prélèvements de cheveux.

\section{SUMMARY}

Exogenous or endogenous androgenic anabolic steroids include 41 substances controlled by the modified appendix of the degree of August $25^{\text {th }}$ march 2005. They also include molecules with similar chemical structure and/or pharmacological effects.

Today, more than 40 active ingredients, representing several tens of patent pharmaceuticals commercialized essentially in the United States, Germany, Italy, Holland, England and Belgium, have been listed in the Biam guide.

In France, a lot of pharmaceuticals containing these active ingredients have been released from the market but can still be purchased from the Internet.

Anabolic steroid are mainly used by athletes to increase their muscle and bones masses, but also to increase their aggressiveness and reduce their recovery time between heavy work. Unfortunately, when used to increase performance, they also present many adverse effects such as imitability; atrophy of genital organs (testicles, ovaries), induction of hormonal dependant cancers (breast, testicles or prostate cancer),...

As for other drugs, there is a major interest to measure these performance enhancing drugs in biological samples for clinical, sport or forensic cases.

The French law conceming the protection of athlete's health indicates that controls can be done using hair samples.

The aim of this work is to present the analytical developments in relation to the dosage of anabolic steroids in unconventional biological specimens, i.e hair; and to evaluate their potential applications.

The main analytical procedure includes a decontamination of a strand of hair; followed by its hydrolysis (solution in 
Ce travail a pour objectif, entre autres, de présenter les développenzents analytiques relatifs au dosage des anabolisants stéroüdiens dans une matrice biologique non conventionnelle, le cheveu, afin d'en évaluer les applications potentielles.

Le tronc analytique commun comprend une décontamination de la mèche de cheveux, son hydrolyse (dissolution dans de la soude ou incubation dans du méthanol), une purification des analytes (extraction sur cartouche et/ou par solvants organiques) et une dérivation de l'extrait avant analyse par chronatographie en phase gazeuse couplée à la spectrométrie de masse tandent. L'utilisation de détecteurs à haute sensibilité et spécificité, précédée parfois d'une techniquue de purification poussée des échantillons, est le pré-requis analytique incontoumable pour la mesure de très faibles concentrations d'anabolisants (de l'ordre du pg/mg) présents dans les cheveux.

\section{MOTS-CLÉS}

Stéroüdes anabolisants, urine, cheveux, dopage, spectrométrie de masse.

\section{Introduction}

Les stéroïdes anabolisants sont, pour la plupart, des dérivés synthétiques de la testostérone (fig. 1). Il s'agit de l'hormone sexuelle mâle synthétisée, par les cellules de Leydig dans les testicules. Cette hormone est aussi produite par les femmes mais en quantité beaucoup plus faible.

La testostérone a plusieurs fonctions importantes pour l'homme (1). Elle possède des effets anabolisants, c'est à dire permettant la biosynthèse des protéines et ainsi entraînant un accroissement rapide de la musculature, une formation accrue des globules rouges et une régénération plus rapide en cas de blessure ou de pathologie.

Elle possède aussi des effets androgéniques qui assurent le développement des organes sexuels mâles ainsi que les caractères sexuels secondaires, comme le système pileux, la mue de la voix, la sécrétion plus ou moins importante de sébum, le développement du pénis, l'agressivité, ou encore la libido.

De plus, elle effectue une rétro-inhibition de sa propre synthèse lorsqu'elle se retrouve en quantité importante dans le sang.

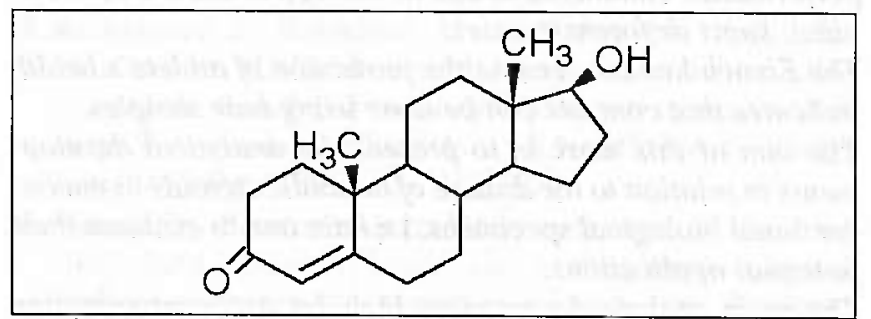

Figure 1 : Structure chimique de la testostérone. sodium carbonate or incubation in methanol), purification of the analytes (extraction on a cartridge and/or by organic solvents) and a derivation of the extract before analysis by gas chromatography coupled with tandem mass spectrometry. The use of high sensitivity and specific detectors before an advanced sample purification is required for the measurement of a very low concentration of anabolic steroids (pg/mg) in hair.

\section{KEY-WORDS}

Anabolic steroids, urine, hair; chronic use, doping, tandem mass spectrometry.

\section{Usage thérapeutique}

Les stéroïdes anabolisants sont des molécules qui ont été mises au point par des laboratoires pharmaceutiques dans un but médical, pour altérer l'équilibre entre les effets anabolisants et androgéniques de la testostérone et ainsi minimiser les effets masculinisants de cette molécule au profit du développement musculaire.

En France, seuls 6 médicaments à base de stéroïdes anabolisants sont inscrits à la Pharmacopée (Tableau I). Ces médicaments soignent des pathologies qui sont principalement dues à des dysfonctionnements hormonaux (2).

Parmi celles-ci, on retrouve des maladies typiquement masculines telles que l'hypogonadisme qui est une affection caractérisée par une fabrication insuffisante d'hormones génitales ou bien la gynécomastie qui conduit à une hypertrophie de la glande mammaire.

Chez les femmes, l'endométriose peut être soignée par les stéroïdes anabolisants, il s'agit d'une maladie gynécologique, entraînant la présence de fragment à l'intérieur de l'utérus (endomètre) en dehors de leur localisation normale, ce qui a pour conséquence la formation de lésions bien délimitées, de type sanguinolentes.

Parmi les maladies pouvant toucher aussi bien les hommes que les femmes, les stéroïdes anabolisants ont un rôle dans le traitement de l'aplasie médullaire (maladie du sang caractérisée par la raréfaction de la moelle osseuse, ce qui a pour conséquence une diminution des globules rouges, des globules blancs et des plaquettes), du lichen scléroatrophique (maladie de peau chronique touchant également les muqueuses des parties génitales) et de l'œdème angioneurotique 
Tableau I: Liste des médicaments contenant des anabolisants et commercialisés en France.

\begin{tabular}{|c|c|l|}
\hline Nom du médicament & Principe actif & \multicolumn{1}{c|}{ Indications } \\
\hline Andractin $^{\odot}$ & Dihydrotestostérone & $\begin{array}{l}\text { Homme : Hypogonadisme par déficit de testostérone } \\
\text { Gynécomastie idiopathique, Lichen scléroatrophique } \\
\text { Femme }: \text { lichen scléroatrophique vulvaire }\end{array}$ \\
\hline Androgel $^{\odot}$ & Testostérone & Hypogonadisme masculin par déficit de testostérone \\
\hline Androtardyl $^{\otimes}$ & Testostérone & Hypogonadisme masculin par déficit de testostérone \\
\hline Danatrol $^{\odot}$ & Danazol & $\begin{array}{l}\text { Endométriose } \\
\text { Eedème angioneurotique héréditaire }\end{array}$ \\
\hline Nilevar $^{\odot}$ & Noréthandrolone & Aplasie médullaire \\
\hline Pantestone $^{\odot}$ & Testostérone undecanoate & Hypogonadisme masculin par déficit de testostérone \\
\hline
\end{tabular}

(maladie conduisant à la formation d'une collection d'eau située dans le derme (partie profonde de la peau), dans l'épiderme (couche superficielle de la peau) ou dans les muqueuses de certains viscères).

En France, 7 médicaments contenant des substances anabolisantes ont été retirés du marché ces dernières années, pour des raisons commerciales et suite au détournement des médicaments pour un usage non thérapeutique (3) ; il s'agit du Dianabol ${ }^{\oplus}$ (métandienone), du Neosteron ${ }^{\circledR}$ (methandriol), du Nastenon ${ }^{\circledast}$. (oxymetholone), du Lontanyl ${ }^{\circledR}$ (testostérone cyclohexylmethylcarbonate), du Sterandryl ${ }^{\oplus}$ retard (testostérone hexahydrobenzoate), de l'Estandron ${ }^{\circledast}$ (testostérone isocaproate) et du Sterandryl ${ }^{\circledR}$ (testostérone propionate) (4). Certains de ces médicaments sont toujours en vente dans d'autres pays et parfois sous d'autres dénominations.

Le faible nombre de médicaments contenant des stéroïdes anabolisants en France n'est pas représentatif du nombre de produits toujours en vente dans les autres pays. Selon le guide Biam, environ 40 anabolisants représentent plusieurs spécialités pharmaceutiques. Ces substances sont surtout commercialisées aux EtatsUnis, aux Pays-Bas, en Allemagne, en Angleterre, en Suisse, en Italie et en Belgique.

Ces médicaments sont principalement utilisés pour soigner l'hypogonadisme masculin, l'aplasie médullaire et certains sont utilisés dans le traitement contre le VIH (5). L'utilisation de ces substances n'est pas sans conséquence, puisqu'elles ont des effets bénéfiques contrebalancés par toute une série d'effets secondaires.

Dans son article, Wu (6) résume les effets secondaires dus à une prise importante de stéroïdes anabolisants.

Tout d'abord chez la femme, ces molécules ont un effet virilisant qui se traduit par de l'hirsutisme (densité excessive de poils dans des zones qui ne sont pas poilues normalement), de l'alopécie (chute de cheveux sur tout ou partie du cuir chevelu) et de l'acné. Dans certains cas, ces effets peuvent être irréversibles.
Chez les hommes, on observe une diminution du volume des testicules, certaines formes de stérilité (réversible), une augmentation de la libido, une diminution de l'HDL-cholestérol, une augmentation de risques de pathologies prostatiques et une gynécomastie.

Au niveau cardiovasculaire, des cardiomyopathies, des infarctus aigus du myocarde, des accidents vasculaires cérébraux et des embolies pulmonaires ont été diagnostiqués à la suite de traitements aux anabolisants.

Au niveau du foie, les anabolisants peuvent être la cause d'effets secondaires importants tels que des tumeurs, des ictères ou une péliose hépatique (kystes remplis de sang dans le foie).

Le but premier des industries pharmaceutiques était d'obtenir des molécules n'ayant plus les effets androgéniques de la testostérone mais à ce jour, il n'en existe aucune, ce qui explique le nombre important d'effets secondaires dus à l'utilisation de ces substances.

La distribution de ces produits aux frontières de la France ainsi que l'explosion des sites Internet consacrés « à la préparation du corps » (7) font qu'il est aujourd'hui très facile de se procurer ces produits et pour un athlète d'avoir recours au dopage.

\section{Usage détourné des sté- roïdes anabolisants}

En effet, les anabolisants, synthétisés dans un but thérapeutique, ont été détournés de leur but premier par le milieu sportif principalement pour leurs effets sur l'augmentation de la masse musculaire et de la matrice osseuse (effets anabolisants) mais aussi parce qu'ils permettent l'augmentation de l'agressivité (8) (important lors des compétitions) et la réduction du temps de récupération entre les efforts.

Le dopage a prịs une place importante dans le milieu du sport aussi bien au niveau amateur que professionnel (9). 
Le dopage est défini comme l'emploi de substances interdites, destinées à accroître provisoirement les capacités physiques de quelqu'un (10).

Afin de faire face au dopage, l'AMA (Agence Mondiale Antidopage, en anglais WADA : World Antidoping Agency) a établi un code mondial antidopage, une liste des substances interdites ainsi que des contrôles lors des compétitions (11).

En France, le ministère de la santé et des sports a publié un arrêté fixant la liste des substances dopantes et méthodes de dopage interdites (identique à celle de l'AMA).

Les stéroïdes anabolisants androgènes, qu'ils soient exogènes (substance qui ne peut pas être produite naturellement par l'organisme humain) ou endogènes (substance produite naturellement par l'organisme humain), comptent 64 substances réglementées par l'arrêté du 25 mars 2005, mais incluent aussi les molécules dont la structure chimique et/ou les effets pharmacologiques sont similaires (12). Cette liste a été mise au point pour répondre à deux préoccupations bien distinctes :

- la protection de la santé des sportifs d'une part,

- la protection de l'éthique et de l'esprit sportif d'autre part.

Dans le cas d'un stéroïde anabolisant pouvant être produit de façon endogène, un échantillon urinaire sera considéré comme positif si la concentration de cette substance ou de ses métabolites, ou de ces marqueurs s'écarte des valeurs normales retrouvées chez l'homme (11). Par exemple, un échantillon sera considéré comme positif lorsque le rapport Testostérone/Epitestostérone (isomère de la testostérone) est supérieur à 4, lorsque la concentration en épitestostérone est supérieure à $200 \mathrm{ng} / \mathrm{ml}$ ou lorsque la concentration d'un des métabolites urinaires de la nandrolone (la 19-norandrostérone : NA) est supérieure à $2 \mathrm{ng} / \mathrm{ml}$ pour un homme ( $5 \mathrm{ng} / \mathrm{ml}$ pour une femme) (11). La présence d'un stéroïde anabolisant exogène dans un échantillon conduira automatiquement à un résultat positif.

Les anabolisants sont des substances interdites depuis les Jeux Olympiques de Munich en 1972. Mais ce n'est qu'en 1976, aux Jeux Olympiques de Montréal, que furent mis en place les premiers contrôles antidopage pour les anabolisants (3). Neuf cas positifs au Dianabol@ (métandienone) y furent identifiés.

Tous les ans, l'AMA publie les statistiques concernant les contrôles anti-dopage, mais ceux de 2005 ne sont pas encore accessibles. En 2004, les anabolisants étaient les prođuits dopants les plus utilisés ( $36 \%$ des cas surtout avec la testostérone, la nandrolone et le stanozolol) suivi par les glucocorticoïdes (16,6\% des cas) (11).

Le CPLD (Conseil de Prévention et de Lutte contre le Dopage) rapporte les infractions commises entre 2000 et 2005 en fonction du sport et de la substance identifiée dans les échantillons (9), le tableau II révèle les anabolisants les plus utilisés et les sports les plus touchés.

Un traitement à base uniquement d'anabolisants ne va pas permettre aux sportifs d'obtenir les résultats escomptés. En effet, l'efficacité des anabolisants dépend également de l'hygiène de vie du sportif. L'entraînement contribue de façon importante aux effets des stéroïdes anabolisants, de même que l'alimentation joue un rôle prépondérant ; la prise de testostérone associée à un régime protéique permettra une augmentation du poids alors qu'avec un régime pauvre en lipides et en protéines le poids restera stable. De plus, les prédispositions de chacun ainsi que le psychisme affecteront plus ou moins les effets des sté-

Tableau II : Nombre de cas positifs aux anabolisants en fonction de la molécule et du sport (de 2000 à 2005) selon le CPLD.

\begin{tabular}{|c|c|c|c|c|c|c|c|}
\hline Sports & Testostérone & Stanozolol & NA + NE & Nandrolone & Métandienone + métabolites & Méténolone & Mesterolone \\
\hline Athlétisme & 0 & 2 & 3 & 1 & 0 & 0 & 0 \\
\hline Aviron & 0 & 0 & 1 & 0 & 0 & 0 \\
\hline Beach-volley & 0 & 0 & 1 & 0 & 0 & 0 & 0 \\
\hline Culturisme & 2 & 7 & 4 & 1 & 0 & 1 & 1 \\
\hline Cyclisme & 4 & 0 & 4 & 1 & 0 & 0 & 0 \\
\hline Football américain & 0 & 0 & 0 & 1 & 0 & 0 & 0 \\
\hline Force athlétique & 1 & 1 & 4 & 1 & 0 & 0 & 0 \\
\hline Haltérophilie & 3 & 2 & 3 & 1 & 1 & 0 & 0 \\
\hline Triathlon & 0 & 0 & 0 & 0 & 0 & 0 \\
\hline Volley-ball & 0 & 0 & 1 & 0 & 6 & 3 & 0 \\
\hline TOTAL & 8 & 12 & 21 & 6 & 0 & 1 \\
\hline
\end{tabular}

La NA (norandrosterone) et la NE (norétiocholanolone) sont des métabolites de la nandrolone. 
roïdes anabolisants. Par exemple, en ce qui concerne le culturisme ou la force athlétique, le sportif utilisera les anabolisants en complément d'un régime protéique pour pouvoir développer ses muscles alors que dans les sports d'endurance, la prise de poids n'étant pas recommandée, le sportif suivra un régime pauvre en lipides et en protéines.

\section{Détection des stéroïdes anabolisants}

Les deux matrices reconnues par l'AMA, sont l'urine et le sang (11). En France, en plus de ces deux matrices, les phanères (poils et cheveux) peuvent être utilisés dans le cadre de la protection de la santé du sportif (Loi Buffet) et d'expertises médico-légales.

\section{Le sang :}

La fenêtre de détection d'une substance dans.le sang est d'environ 1 jour, ce qui ne permet pas d'avoir beaucoup de recul quant à une prise de produits dopants dans les jours qui précèdent le contrôle. De plus la prise de sang est une méthode invasive (10). (méthode d'exploration ou de soins qui provoque des lésions de l'organisme).

Le sang n'est pas utilisé dans les contrôles antidopage en ce qui concerne les anabolisants. En effet, dans le guide antidopage édité pour les Jeux Olympiques de Turin (13), il est indiqué que le sang est utilisé pour le dépistage de l'EPO (en complément de l'urine), de l'hormone de croissance (hGH), de l'hémoglobine de synthèse (HBOC) ou pour dépister les transfusions.

Le prélèvement de sang est effectué par une personne qui possède un certificat de prélèvement. Deux échantillons de sang seront prélevés, d'environ $3 \mathrm{ml}$ chacun (le volume pourra être spécifié par le laboratoire d'analyse). Le sang sera recueilli dans des tubes contenant un anti-coagulant (comme l'EDTA : acide éthylènediaminotétraacétique) (11).

Les échantillons sanguins sont conservés à $+4^{\circ} \mathrm{C}$ avant analyse.

\section{Les urines :}

La fenêtre de détection des substances y est de 2 à 4 jours dans l'urine, excepté pour les esters des stéroïdes anabolisants qui sont décelables pendant 6 à 7 jours. L'urine permet donc de connaître s'il y a eu prise d'anabolisants dans les jours précédents le prélèvement.

L'urine permet de détecter majoritairement les métabolites des stéroïdes anabolisants. Les substances mères quand à elles, peuvent être identifiées mais elles sont en général en concentrations plus faibles.
Dans le cadre des contrôles antidopage organisés par l'AMA, le prélèvement d'urine sera effectué en présence d'un agent de contrôle du dopage (ACD) qui s'assurera de l'intégrité, la validité et l'identité de l'échantillon (11). Le sportif urine dans un gobelet collecteur, l'urine sera ensuite séparée dans deux flacons et l'excédent sera jeté par l'ACD.

Les échantillons urinaires sont conservés à $+4^{\circ} \mathrm{C}$ avant analyse.

L'AMA a accrédité 33 laboratơires de part le monde pour effectuer les analyses anti-dopage à partir des prélèvements effectués lors des compétitions internationales et lors des Jeux Olympiques.

Ces laboratoires sont soumis à l'application de la norme ISO/CEI 17025 pour l'analyse des échantillons de contrôle du dopage. L'AMA a d'ailleurs édité un standard international pour les laboratoires accrédités (11).

Il n'existe pas de méthodes normalisées pour les analyses de contrôle du dopage. Le laboratoire doit développer, valider et documenter ses propres méthodes internes pour l'analyse des substances inscrites à la liste des interdictions et des substances apparentées (11).

Beaucoup de travaux ont été réalisés sur l'identification des stéroïdes anabolisants dans l'urine puisqu'elle est la matrice de choix dans le contrôle antidopage. Par exemple, le métabolite recherché dans les cas de dopage à la nandrolone est la norandrosterone (NA) (14); le seuil minimal pour conclure à un résultat positif est de $2 \mathrm{ng} / \mathrm{ml}$ pour la NA chez l'homme.

Dans le cadre d'une étude, Jeanneau et al. (15) ont déterminé les taux physiologiques de NA et NE à partir d'une soixantaine d'échantillons urinaires provenant de sportifs de haut niveau ou non. Cette étude, comme plusieurs autres, a permis de confirmer le seuil de positivité fixé à $2 \mathrm{ng} / \mathrm{ml}$. En effet, les valeurs obtenues étaient en moyenne inférieures à $0,5 \mathrm{ng} / \mathrm{ml}$ et aucun échantillons ne présentait de valeur supérieure à $1 \mathrm{ng} / \mathrm{ml}$. Cependant, il est à souligner que dans le cas des femmes enceintes, les concentrations obtenues sont souvent supérieures à $2 \mathrm{ng} / \mathrm{ml}$, confirmant ainsi la sécrétion naturelle de 19-norstéroïdes pendant la grossesse.

Une étude multicentrique organisée au sein de la SFTA (Société Française de Toxicologie Analytique) a eu pour but de détermiñer les concentrations physiologiques des métabolites de la nandrolone dans les urines humaines (16). Il a été démontré que les métabolites de la nandrolone, la NA et la NE, sont produits dans un rapport 72/28 avec une concentration inférieure à $2 \mathrm{ng} / \mathrm{ml}$ pour la NA. Cette étude a porté sur un panel de 160 échantillons provenant d'une population témoin incluant des sportifs. Brièvement, la méthode employée était la suivante : après avoir été centrifugés, 
$2,5 \mathrm{ml}$ d'urines sont prélevés et $5 \mathrm{ng}$ de noretiocholanolone $\mathrm{d}_{3}$ utilisés comme standard interne. L'extraction se fait sur colonnes Isolute $\mathrm{C}_{18}$, l'échantillon y est déposé, les colonnes sont lavées à l'eau bidistillée et séchées sous vide. L'élution est faite par ajout de $2 \mathrm{x}$ $0,75 \mathrm{ml}$ de méthanol. L'éluat récupéré est évaporé sous vide avant d'être repris dans $1 \mathrm{ml}$ de tampon phosphate et de $100 \mu \mathrm{l}$ de $\beta$-glucuronidase et incubé à $50^{\circ} \mathrm{C}$ pendant $1 \mathrm{~h}$. Afin de mettre les analytes sous leur forme libre, une étape supplémentaire de purification est effectuée par ajout de $2 \mathrm{ml}$ de pentane puis agitation et centrifugation. La phase organique est ensuite prélevée puis évaporée. La dernière étape consiste en la dérivation de l'extrait sec par un mélange de MSTFA (NMéthyl-N-triméthylsilyltrifluoroacétamide), $\mathrm{NH}_{4} \mathrm{I}$ et 2-mercaptoéthanol.

Les analytes sont ensuite séparés par chromatographie gazeuse et détectés par un spectromètre de masse.

Cette étude a mis en évidence de nouvelles questions quant à la présence de NA dans les urines de sportifs à la suite d'ingestion de viande d'animaux traités avec de la nandrolone ou en sécrétant naturellement, comme dans le cas des sangliers. Cependant, pour dépasser le seuil de positivité fixé, la consommation de fortes quantités de viande (de l'ordre du kilogramme) est nécessaire. Des solutions ont été proposées pour pouvoir différencier l'utilisation de produits dopants d'une consommation importante de viande ; en effet, chez les sportifs dopés, le rapport NA/NE est compris entre 3 et 5 , alors que dans les urines non dopées, il est inférieur à 3 .

La cinétique d'élimination urinaire de la nandrolone, après l'administration de différentes formes, a été publiée en 1999 (14). Lors de l'administration de $22 \mathrm{mg}$ par voie orale de nandrolone, il s'est avéré que la NA est toujours trouvée en concentration plus importante que la NE. Les deux métabolites ont des cinétiques d'élimination identiques : à savoir, une concentration maximale des métabolites au bout de $4 \mathrm{~h}$ (environ $30000 \mathrm{ng} / \mathrm{ml}$ pour la NA et $6000 \mathrm{ng} / \mathrm{ml}$ pour la $\mathrm{NE}$ ) et qui chute jusqu'à ne plus être décelable au bout de $24 \mathrm{~h}$. Par contre lorsque la nandrolone est injectée en intra-musculaire (50 $\mathrm{mg}$ de nandrolone décanoate), les concentrations maximales sont plus faibles (environ $80 \mathrm{ng} / \mathrm{ml}$ pour la NA et $30 \mathrm{ng} / \mathrm{ml}$ pour la NE) et l'élimination est beaucoup plus lente. En effet, 4 mois après l'injection, la concentration de NA dans les urines était encore d'environ $10 \mathrm{ng} / \mathrm{ml}$ et 9 mois après elle avoisinait le seuil de positivité de $2 \mathrm{ng} / \mathrm{ml}$. Selon la forme sous laquelle la nandrolone est administrée, il a été montré que les concentrations urinaires des métabolites et leur élimination n'étaient pas les mêmes.

Dans leur article, Kintz et al. (14) montrent aussi que la NA et la NE peuvent aussi être excrétées dans les urines à la suite d'une administration de 19-norstéroïdes (norandrostenediol et norandrostenedione).

Il est à noter qu'après une prise de norandrostenediol, la NA sera en concentration plus importante que la NE dans les 21 premières heures, puis le rapport s'inversera. Après une prise de norandrostenedione, la NE est-le métabolite majoritaire pendant les 48 premières heures puis le rapport s'inverse.

De nombreux anabolisants sont recherchés, leurs métabolites n'étant pas tous connus. Dans ce cas, on recherche soit la présence de l'anabolisant lorsqu'il est exogène soit si la concentration de l'anabolisant endogène dépasse le seuil de positivité.

Un autre exemple est celui de la testostérone. Afin de savoir s'il y a eu dopage par administration de testostérone ou d'esters de testostérone, les laboratoires mesurent le rapport Testostérone/Epitestostérone (isomère de la testostérone). Jusqu'en 2005, le rapport Testostérone/Epitestostérone, à ne pas dépasser, était de 6. Ce seuil a été harmonisé avec le seuil mentionné dans le document technique des laboratoires antidopage et il a été abaissé à 4 (11).

\section{Les cheveux :}

Contrairement à l'urine, la fenêtre de détection des substances dans les phanères est plus importante. En effet, les xénobiotiques (substances étrangères à l'organisme) ainsi que les substances endogènes, se trouvant dans le sang, vont diffuser vers les cellules en croissance des bulbes pileux. Ces cellules piègent les xénobiotiques dans les structures kératinisées lorsqu'elles fusionnent pour former le poil. Dans certains cas, les xénobiotiques se lient à la mélanine (pigment des cheveux et des poils) permettant ainsi une meilleure incorporation (17).

Ceci permet d'augmenter la fenêtre de détection des xénobiotiques qui passe alors à plusieurs mois en fonction de la longueur du poil. Etant donné que les cheveux poussent en moyenne de $1 \mathrm{~cm}$ par mois, il èst possible d'obtenir une chronologie de la consommation d'un produit. Si ces derniers sont trop courts ou tout simplement absents, les poils pubiens seront utilisés (18). Dans les cas extrêmes, l'utilisation des poils axillaires ou de barbe peut être envisagée.

L'analyse des cheveux permettra de détecter majoritairement les substances mères, leurs métabolites peuvent aussi être incorporés dans les cheveux mais en beaucoup plus faible quantité.

Une standardisation du prélèvement des cheveux consiste à effectuer le prélèvement à la surface de la peau au niveau du vertex postérieur (arrière du crâne). C'est au niveau de cette région que la pousse des cheveux subit le moins de variations selon l'âge, le sexe ou 
la saison.

Une mèche de 60 cheveux (diamètre d'un crayon à papier) est suffisante pour l'analyse. L'orientation de la mèche est nécessaire si une analyse segmentaire est demandée : une cordelette sera fixée à $1 \mathrm{~cm}$ de la racine avant d'être coupée au ciseau. La racine permet de voir une consommation récente et la pointe, une consommation ancienne.

La conservation se fait dans une enveloppe ou un tube sec à température ambiante (19).

Cette matrice possède plusieurs avantages par rapport à l'urine. Tout d'abord, il s'agit d'une méthode de prélèvement non invasive et dont la conservation est aisée.

L'analyse de cheveux permet aussi de faire face au problème de l'adultération. En effet, les sportifs ont développé de nombreuses techniques pour fausser les tests antidopage, en diluant leur urine, en utilisant d'autres urines que les leurs, en ajoutant des substances qui vont dégrader les analytes recherchés, ou en utilisant des agents masquants. Les phanères étant prélevés directement sur la tête ou le corps de la personne, le risque d'adultération par l'athlète est nettement réduit.

Si nécessaire, les cheveux permettent de prélever ultérieurement un second échantillon identique au premier, ce qui est totalement impossible avec l'urine.

A ce jour, les cheveux ne sont toujours pas une matrice reconnue par l'AMA. En France, les cheveux sont utilisés par quelques laboratoires dans des domaines d'activité différente, tels que les affaires médico-légales. L'analyse des cheveux est reconnue par la justice française et peut ainsi être utilisée pour mettre en évidence une prise de GHB (acide gamma hydrobutyrique) ou de zolpidem dans les cas de soumission chimique ; ainsi qu'en médecine du travail dans les cas d'exposition professionnelle à des substances toxiques.

Une telle application a été effectuée par Cirimele et al., dans le cadre de l'interpellation de deux culturistes arrêtés à la frontière avec 2050 comprimés et 251 ampoules de stéroïdes anabolisants (20).

Dans le cas d'usage d'anabolisants, l'analyse des cheveux a un grand intérêt. Il a été rapporté dans plusieurs articles que les sportifs qui utilisent des anabolisants ne le font pas pendant toute l'année. Certains athlètes prennent des anabolisants pendant les périodes hivernales et arrêtent leur traitement pendant les périodes de compétition $(21,22)$. D'autres alternent les périodes de traitement (4 à 18 semaines) et les périodes sans prise d'anabolisants (1 mois par an) (23). Dans ces cas, l'analyse urinaire pourra conduire à un résultat négatif alors que le sportif a bien utilisé un produit dopant (les anabolisants étant des substances interdites pendant et hors compétition).
Thieme et al. (24) ont développé une méthode d'analyse pour détecter le clenbutérol, la métandienone et les esters testostérone, nandrolone et méténolone.

La première étape qui consiste à une extraction méthanolique permet d'isoler, dans un premier temps, les esters chimiquement instables dans des conditions d'hydrolyse telle que la soude.

Les cheveux sont incubés dans du méthanol, qui est repris puis évaporé. Une extraction liquide-liquide est effectuée : le méthanol est évaporé, puis repris dans du tampon à $\mathrm{pH} 9$ et extrait par du diéthyle éther. La phase d'éther est évaporée puis reprise dans une solution d'acétonitrile/eau $(5 / 95, \mathrm{v} / \mathrm{v})$. Une HPLC préparative est effectuée avant la dérivation et l'injection en GCHRMS et GC-MS/MS.

L'extraction des autres anabolisants est effectuée par dissolution des cheveux dans la soude, étape de SPE (Solid phase extraction), extraction liquide-liquide et dérivation. L'agent dérivant utilisé est un mélange de MSTFA/ $/ \mathrm{NH}_{4} \mathrm{I} /$ dithioerythrol.

Gaillard et al. (22) utilisent une méthode d'extraction différente de celle mentionnée précédemment. La première étape, après la dissolution des cheveux, consiste en une extraction sur colonnes de $\mathrm{NH}_{2}$ activée par $3 \mathrm{ml}$ d'acétate d'éthyle. L'élution s'effectue aussi avec l'acétate d'éthyle, les stéroïdes n'étant pas retenus sur cette colonne. L'éluat est ensuite évaporé avant d'être repris dans $1 \mathrm{ml}$ de chloroforme.

Une deuxième étape de purification est effectuée sur des colonnes de silice activée par du chloroforme. Les esters sont récupérés après le passage de l'échantillon et une élution avec un mélange de chloroforme/acétate d'éthyle $(3: 1, v . / v)$ est nécessaire pour les stéroïdes anabolisants. Après évaporation, l'extrait sec est dérivé par un mélange de $\mathrm{MSTFA} / \mathrm{NH}_{4} \mathrm{I} / 2$-mercaptoéthanol 20 min à $80^{\circ} \mathrm{C}$ et analysé par chromatographie en phase gazeuse couplée à un spectromètre de masse en tandem. Cette méthode permet d'identifier les molécules suivantes : la nandrolone, l'estradiol, la testostérone, la boldenone, la méthyltestostérone, l'éthynilestradiol, la métandienone, la testostérone propionate, phénylpropionate, cypionate, décanoate et undécanoate ainsi que la nandrolone décanoate.

Les cheveux peuvent se révéler-intéressants dans le cadre d'une expertise judiciaire de contrôle du dopage. Récemment, à la suite d'une demande d'un sportif déclaré positif à la métandienone après un contrôle antidopage, une analyse segmentaire a été effectuée un mois plus tard afin de savoir s'il s'agissait d'un cas de dopage à l'insu du sportif (25).

Une méthode a été développée pour la détection d'un grand nombre de stéroïdes anabolisants et de leurs 
esters (Tableau $\mathrm{III}$ ). La méthode d'extraction des esters change légèrement de celle des stéroïdes anabolisants, il s'agit de la procédure décrite par Kintz et al. (26). Le changement majeur est une détection par spectrométrie de masse en tandem.

Le tableau III présente les ions parents, ions fils, temps de rétention et énergies de collision, optimisés pour ces différents stéroïdes anabolisants.

Chaque molécule analysée est identifiée grâce à son temps de rétention (spécifique) ainsi qu'aux transitions de quantification et d'identification de cette molécule. La quantification est réalisée à l'aide d'une gamme de calibrateurs préparée en présence de cheveux témoins surchargés.

Dans un premier temps, les cheveux de ce sportif ont été coupés en quatre segments de $1 \mathrm{~cm}$ chacun après décontamination dans deux bains successifs de dichlorométhane. Les cheveux ont ensuite été dissous dans de la soude, en présence de nandrolone $d_{3}$ utilisé comme étalon interne. L'homogénat a ensuite été neutralisé par ajout d'acide chlorhydrique et de tampon phosphate.

Une première étape d'extraction des anabolisants est effectuée sur colonne Isolute $\mathrm{C}_{18}$ qui $\mathrm{a}$, au préalable, été conditionnée avec du méthanol puis de l'eau bidistillée. Après dépôt de l'échantillon, les colonnes sont lavées à

Tableau III : Paranètres de chromatographie et de spectrométrie de masse pour la méthode de screening des anabolisants dans les cheveux.

\begin{tabular}{|c|c|c|c|c|}
\hline Substance & $\begin{array}{c}\text { Temps de } \\
\text { rétention } \\
\text { (min) }\end{array}$ & $\begin{array}{c}\text { Ion parent } \\
(\mathrm{m} / \mathrm{z})\end{array}$ & $\begin{array}{c}\text { Ion fils } \\
(\mathrm{m} / \mathrm{z})\end{array}$ & $\begin{array}{l}\text { Energie de } \\
\text { collision } \\
(\mathrm{eV})\end{array}$ \\
\hline Testostérone & 9,68 & 432,4 & 209,2 & 10 \\
\hline Epitestostérone & 9,42 & 432,4 & 209,2 & 10 \\
\hline DHEA & 9,30 & 432,4 & 327,3 & 10 \\
\hline Testostérone $\mathrm{d}_{3}$ & 9,67 & 435,4 & 420,4 & 10 \\
\hline Nandrolone & 9,45 & 418,4 & 194,2 & 10 \\
\hline Nandrolone $d_{3}$ & 9,44 & 421,4 & 194,2 & 15 \\
\hline Metenolone & 9,84 & 195,1 & 179,0 & 10 \\
\hline Mesterolone & 9,62 & 448,3 & 141,1 & 15 \\
\hline 19-Norandrostenédione & 9,33 & 416,3 & 234,2 & 10 \\
\hline 19-Norandrostenediol & 9,13 & 240,2 & 225,2 & 5 \\
\hline & & & & \\
\hline Testostérone acétate & 12,44 & 402,4 & 209,1 & 10 \\
\hline Testostérone propionate & 12,82 & 416,3 & 209,1 & 10 \\
\hline Testostérone isocaproate & 13,80 & 458,4 & 209,2 & 15 \\
\hline Testostérone énanthate & 14,38 & 472,4 & 209,2 & 10 \\
\hline Testostérone benzoate & 15,49 & 484,4 & 209,2 & 15 \\
\hline Testostérone cypionate & 15,29 & 464,3 & 209,1 & 20 \\
\hline Testostérone phénylpropionate & 16,30 & 492,3 & 477,3 & 10 \\
\hline Testostérone décanoate & 16,06 & 514,5 & 209,2 & 10 \\
\hline Metenolone énanthate & 14,57 & 486,3 & 208,1 & 10 \\
\hline Nandrolone décanoate & 15,76 & 500,4 & 194,2 & 15 \\
\hline Nandrolone phénylpropionate & 15,97 & 478,3 & 182,2 & 15 \\
\hline
\end{tabular}

l'eau bidistillée puis séchées sous vide. L'élution se fait avec 3 fois $0,5 \mathrm{ml}$ de méthanol. L'éluat ainsi récupéré est évaporé sous vide, puis repris dans $1 \mathrm{ml}$ de tampon phosphate.

Une deuxième étape d'extraction est effectuée par addition de $2 \mathrm{ml}$ de pentane et $100 \mathrm{mg}$ du mélange $\mathrm{Na}_{2} \mathrm{CO}_{3}-\mathrm{NaHCO}_{3}(1: 10)$. Après agitation et centrifugation, la phase organique est évaporée.

Le résidu est ensuite dérivé par l'addition de MSTFA et du mélange MSTFA- $\mathrm{NH}_{4} \mathrm{I}-2$-mercaptoéthanol pendant $20 \min$ à $60^{\circ} \mathrm{C}$.

L'analyse a ensuite été effectuée sur un GC-MS/MS. Un aliquot de $1 \mu \mathrm{l}$ de l'extrait dérivé a été injecté en mode sans division dans une colonne d'un chromatographe Hewlett Packard (6890 Series). Le débit du gaz vecteur (hélium, pur à 99.9999\%) à travers la colonne (colonne capillaire HP5-MS, 5\% phenol-95\% méthylsiloxane, $30 \mathrm{~m} \times 0,32 \mathrm{~mm}$ i.d., $0,25 \mu \mathrm{m}$ ) était de $1,5 \mathrm{ml} / \mathrm{min}$. La température de l'injecteur est de $270^{\circ} \mathrm{C}$. La température a été programmée pour augmenter de $30^{\circ} \mathrm{C} / \mathrm{min}$ jusqu'à $295^{\circ} \mathrm{C}$ en commençant à $60^{\circ} \mathrm{C}$; la température finale maintenue $10 \mathrm{~min}$.

La détection a été réalisée par un détecteur de masse en tandem Waters Quattro Micro opérant en impact électronique à $70 \mathrm{eV}$ et les données ont été enregistrées selon le mode multiple reaction monitoring (MRM). Les ions parents, ions fils, temps de rétention et énergies de collision, optimisés pour la métandienone et la nandrolone $\mathrm{d}_{3}$, sont présentés dans le Tableau IV.

A travers l'analyse d'une mèche de cheveux, il a été possible de confirmer l'exposition à la métandienone avec l'information supplémentaire que cette consommation existait depuis plus 4 mois étant donné la présence de métandienone dans les 4 segments de cheveux à des concentrations variant entre 7 et $108 \mathrm{pg} / \mathrm{mg}$ de cheveux. Les figures 2 et 3 représentent respectivement les chromatogrammes du calibrateur à $15 \mathrm{pg} / \mathrm{mg}$ et celui obtenu à partir du premier segment de cheveux du sportif $(0$ à $1 \mathrm{~cm})$.

Il est à noter que les cheveux de ce sportif étaient décolorés, ce qui signifie que les quantités de substance dopante trouvées sont inférieures à celles qui auraient

Tableau IV : Paramètres de chromatographie et de spectrométrie de masse pour la métandienone.

\begin{tabular}{|c|c|c|c|c|}
\hline Substance & $\begin{array}{c}\text { Temps de } \\
\text { rétention }(\mathbf{m i n})\end{array}$ & $\begin{array}{c}\text { Ion parent } \\
(\mathbf{m} / \mathbf{z})\end{array}$ & $\begin{array}{c}\text { Ions fils } \\
(\mathbf{m} / \mathbf{z})\end{array}$ & $\begin{array}{c}\text { Energie de } \\
\text { collision }(\mathbf{e V})\end{array}$ \\
\hline \multirow{2}{*}{ Métandiénone } & & & 339,2 & 10 \\
\cline { 4 - 5 } & \multirow{2}{*}{8,96} & 444,2 & 206,1 & 15 \\
\cline { 4 - 5 } & & 206,1 & 191 & 10 \\
\hline Nandrolone $\mathrm{d}_{3}$ & 8,50 & 421,4 & 194,2 & 15 \\
\hline
\end{tabular}


Annales de Toxicologie Analytique, vol. XVIII, $n^{\circ} 1,2006$

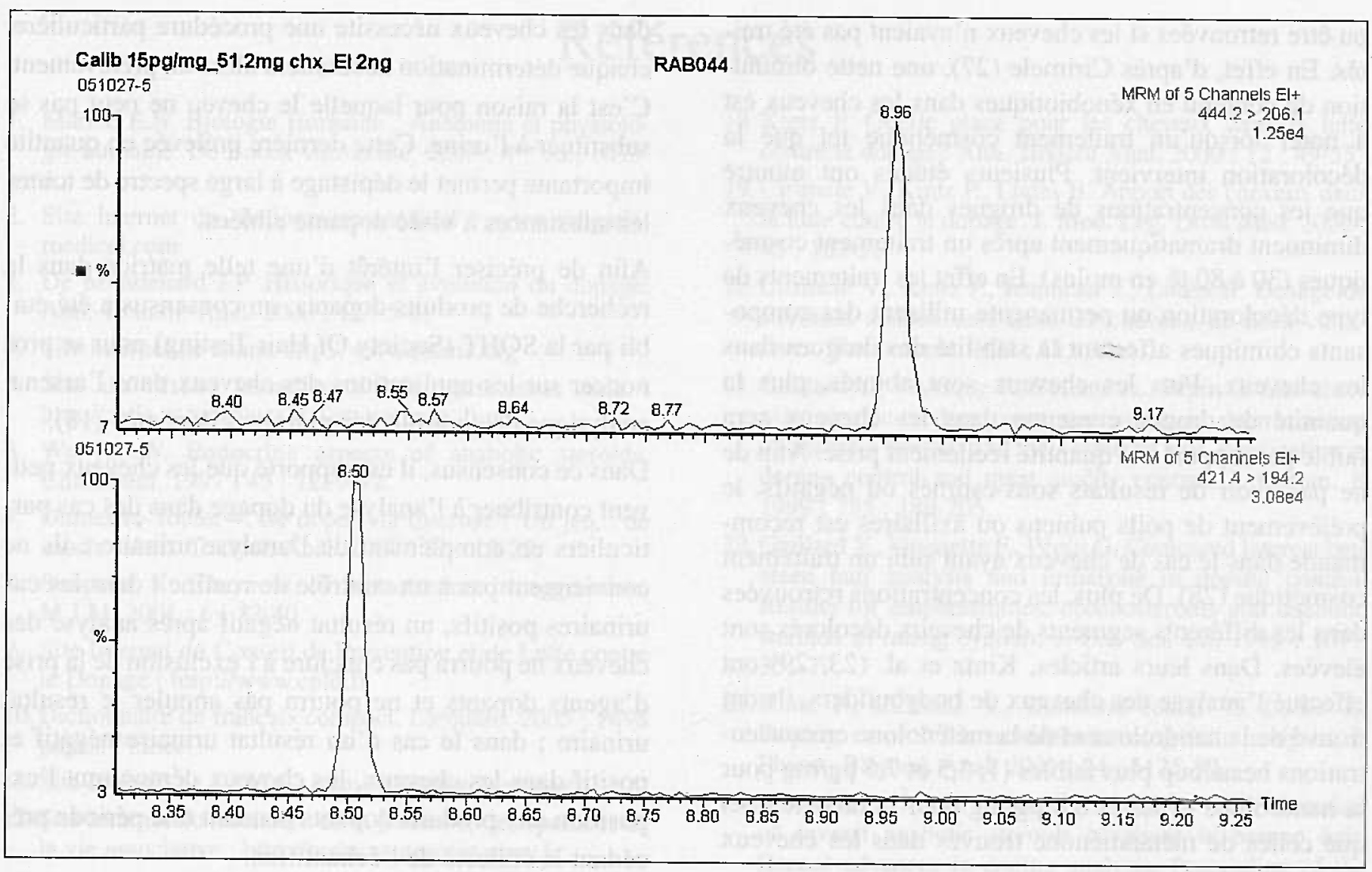

Figure 2 : Chromatogramme obtenu à partir de $51,2 \mathrm{mg}$ de cheveux dopés à une concentration funale de $15 \mathrm{pg} / \mathrm{mg}$. Haut : transition de quantification $\mathrm{m} / \mathrm{z}$ ion parent $444,2, \mathrm{~m} / \mathrm{z}$ ion fils 206,1 . Bas : nandrolone $d_{3}$ avec son ion flls $\mathrm{m} / \mathrm{z}, 194,2$.

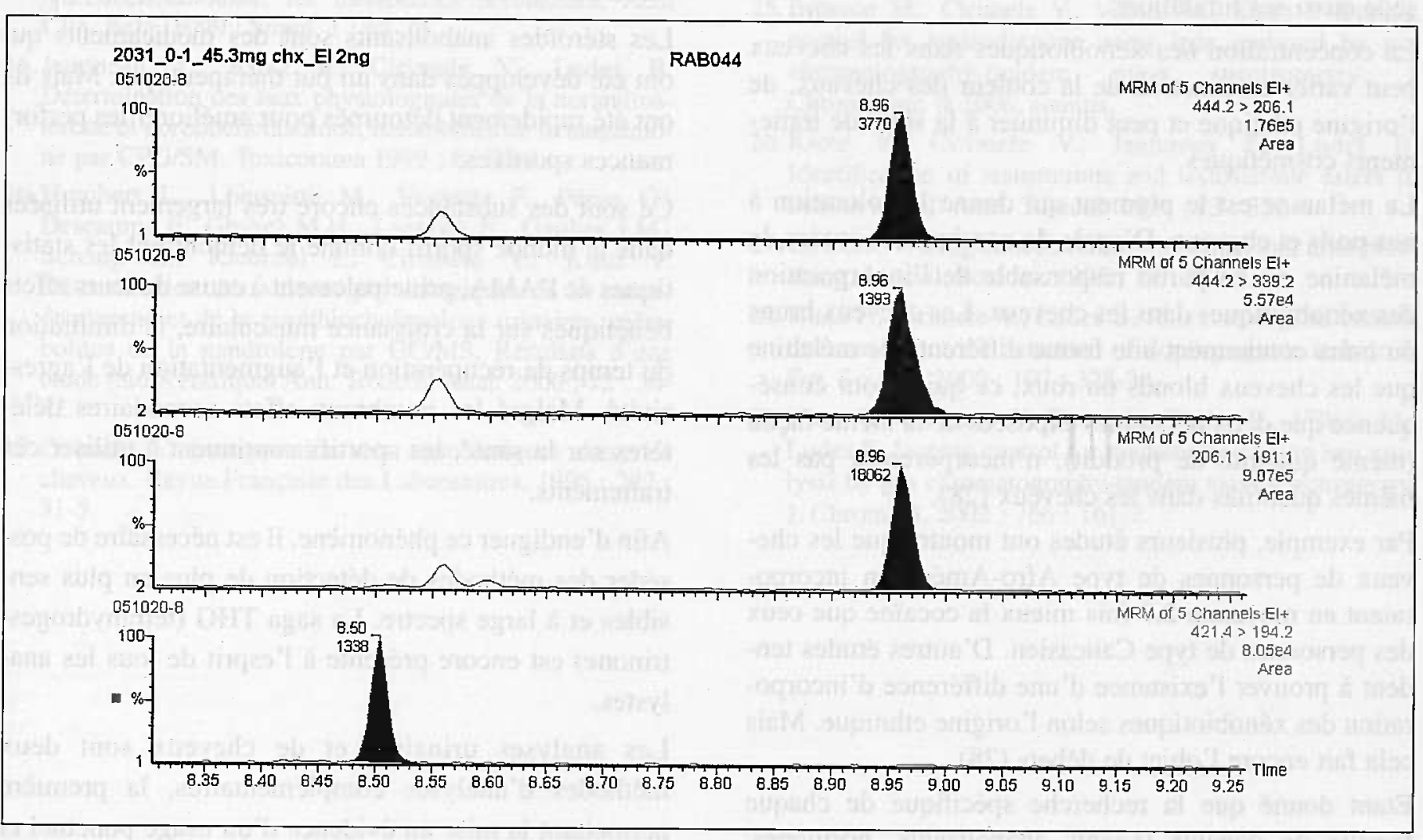

Fïgure 3 : Chromatogramme obtenu à partir de $45,3 \mathrm{mg}$ de cheveux du sportif. La métandienone a été quantifiée à la concentration de $79,1 \mathrm{pg} / \mathrm{mg}$. Haut : transition de quantification pour la métandiénone. Milieux : 2 transitions d'identification de la métandienone. Bas : nandrolone $d_{3}$ avec son ion fils $\mathrm{m} / \mathrm{z}$ 194,2. 
pu être retrouvées si les cheveux n'avaient pas été traités. En effet, d'après Cirimele (27), une nette diminution du contenu en xénobiotiques dans les cheveux est à noter lorsqu'un traitement cosmétique tel que la décoloration intervient. Plusieurs études ont montré que les concentrations de drogues dans les cheveux diminuent dramatiquement après un traitement cosmétiques ( 30 à $80 \%$ en moins). En effet les traitements de type décoloration ou permanente utilisent des composants chimiques affectant la stabilité des drogues dans les cheveux. Plus les cheveux sont abîmés, plus la quantité de drogue contenue dans les cheveux sera faible par rapport à la quantité réellement prise. Afin de ne pas avoir de résultats sous-estimés ou négatifs, le prélèvement de poils pubiens ou axillaires est recommandé dans le cas de cheveux ayant subi un traitement cosmétique (28). De plus, les concentrations retrouvées dans les différents segments de cheveux décolorés sont élevées. Dans leurs articles, Kintz et al. $(23,29)$ ont effectué l'analyse des cheveux de bodybuilders, ils ont trouvé de la nandrolone et de la méténolone en concentrations beaucoup plus faibles (1, 3,5 et 7,5 $\mathrm{pg} / \mathrm{mg}$ pour la nandrolone et 7,3 et $8,8 \mathrm{pg} / \mathrm{mg}$ pour la méténolone) que celles de métandienone trouvés dans les cheveux de ce sportif.

La matrice cheveu ne présente pas que des avantages par rapport à l'urine ; comme chaque matrice, elle possède aussi ses limitations.

La concentration des xénobiotiques dans les cheveux peut varier en fonction de la couleur des cheveux, de l'origine ethnique et peut diminuer à la suite de traitements cosmétiques.

La mélanine est le pigment qui donne la coloration à nos poils et cheveux. D'après de nombreuses études, la mélanine est en partie responsable de l'incorporation des xénobiotiques dans les cheveux. Les cheveux bruns ou noirs contiennent une forme différente de mélanine que les cheveux blonds ou roux; ce qui a pour conséquence que deux personnes exposées de la même façon (même quantité de produit), n'incorporeront pas les mêmes quantités dans les cheveux (28).

Par exemple, plusieurs études ont montré que les cheveux de personnes de type Afro-Américain incorporaient en moyenne 2,9 fois mieux la cocaïne que ceux des personnes de type Caucasien. D'autres études tendent à prouver l'existence d'une différence d'incorporation des xénobiotiques selon l'origine ethnique. Mais cela fait encore l'objet de débats (28).

Etant donné que la recherche spécifique de chaque famille de dopants (agents anabolisants, hormones, agents avec activité anti-œstrogène, diurétiques, stimulants, narcotiques, cannabinoïdes et glucocorticoïdes) dans les cheveux nécessite une procédure particulière, chaque détermination nécessitera alors un prélèvement. C'est la raison pour laquelle le cheveu ne peut pas se substituer à l'urine. Cette dernière prélevée en quantité importante permet le dépistage à large spectre de toutes les substances à visée dopante ciblées.

Afin de préciser l'intérêt d'une telle matrice dans la recherche de produits dopants, un consensus a été établi par la SOHT (Society Of Hair Testing) pour se prononcer sur les applications des cheveux dans l'arsenal analytique de contrôle des pratiques dopantes (18).

Dans ce consensus, il est rapporté que les cheveux peuvent contribuer à l'analyse du dopage dans des cas particuliers en complément de l'analyse urinaire; ils ne conviennent pas à un contrôle de routine ; dans les cas urinaires positifs, un résultat négatif après analyse des cheveux ne pourra pas conclure à l'exclusion de la prise d'agents dopants et ne pourra pas annuler le résultat urinaire ; dans le cas d'un résultat urinaire négatif et positif dans les cheveux, les cheveux démontrent l'exposition aux produits dopants pendant une période précédant la collecte de l'échantillon.

\section{Conclusion}

Les stérö̈des anabolisants sont des médicaments qui ont été développés dans un but thérapeutique. Mais ils ont été rapidement détournés pour améliorer les performances sportives.

Ce sont des substances encore très largement utilisées dans le monde sportif comme le démontrent les statistiques de $1^{\prime} \mathrm{AMA}$, principalement à cause de leurs effets bénéfiques sur la croissance musculaire, la diminution du temps de récupération et l'augmentation de l'agressivité. Malgré les nombreux effets secondaires délétères sur la santé, les sportifs continuent à utiliser ces traitements.

Afin d'endiguer ce phénomène, il est nécessaire de posséder des méthodes de détection de plus en plus sensibles et à large spectre. La saga THG (tétrahydrogestrinone) est encore présente à l'esprit de tous les analystes.

Les analyses urinaires et de cheveux sont deux méthodes d'analyses complémentaires, la première permettant la mise en évidence d'un usage ponctuel et la seconde un usage répété. Il serait temps que cette association soit reconnue par l'AMA. 


\section{Références}

1. Marieb E.N. Biologie Humaine : Anatomie et physiologie humaine. De Boeck Université. 2000 ; $4^{\text {ine }}$ ed., 10534.

2. Site internet du dictionnaire médical : www.vulgarismedical.com

3. De Mondenard J.P. Historique et évolution du dopage. Ann. Toxicol. Anal. $2000 ; 12: 5-8$.

4. Site internet de Biam: http://www.biam2.org

5. Site du réseau canadien d'info traitements sida: http://catia.ca/pdf/facts8f/Testosterone_fr.pdf

6. Wu F.C.W. Endocrine aspects of anabolic steroids, Clin.Chem. $1997 ; 43: 1289-92$.

7. Dumestre-Toulet V. Se doper via Internet ? Un jeu... de souris ! Ann. Toxicol. Anal. $2000 ; 12$ : 19-25.

8. Simpson K. The role of testosterone in aggression. M.J.M. $2001 ; 6: 32-40$.

9. Site Internet du Conseil de Prévention et de Lutte contre le Dopage : http://www.cpld.fr

10. Dictionnaire de français compact. Larousse. $2005 ; 1498$ pages, France.

11. Site Internet de l'AMA : http://www.wada-ama.org

12. Site Internet du ministère de la jeunesse, des sports et de la vie associative : http://www.santesport.gouv.fr

13. Guide antidopage édité pour les JO de Turin : http://turin.franceolympique.com

14. Kintz P., Cirimele V., Ludes B. Norandrosterone et Noretiocholanolone: les métabolites révélateurs, Acta Clin. Belg. 1999 ; Suppl. 1 : 68-73.

15. Jeanneau T., Kintz P., Cirimele V., Ludes B. Détermination des taux physiologiques de la norandrosterone et noretiocholanolone, métabolites de la nandrolone par CPG/SM. Toxicorama $1999 ; 6: 25-9$.

16. Humbert L., Lhermitte M., Vaysette F., Pépin G., Descamps F., Ghysel M.H., Lacassie E., Gaulier J.M., Schang L., Ricordel I., Cirimele V., Kintz P. Détermination des valeurs physiologiques de la norandrosterone et de la noréthiocholanolone urinaires, métabolites de la nandrolone par GC/MS. Résultats d'une étude multicentrique. Ann. Toxicol. Anal. 2000 ; 12 : 3642.

17. Cirimele V. Incorporation des xénobiotiques dans les cheveux. Revue Française des Laboratoires. 1996 ; 282 : 31-5.
18. Kintz P. Quelle place pour les cheveux dans la lutte contre le dopage? Ann. Toxicol Anal. $2000 ; 12: 49-55$.

19. Cirimele V., Kintz P., Ludes B. Apport des cheveux dans la lutte contre le dopage. J. Med. Leg. Droit Med. 2000 ; $43: 187-91$.

20. Cirimele V., Kintz P., Jeanneau T., Ludes B. Dosage de stéroïdes anabolisants dans les cheveux de deux culturistes. Toxicorama $1999 ; 11: 30-4$.

21. Gaillard Y., Vayssette F., Balland A., Pépin G. Gas-chromatographic-tandem mass spectrometric determination of anabolic steroids and their esters in hair. Application in doping control and meat quality control. J. Chrom. B $1999 ; 735: 189-205$.

22. Gaillard Y., Vayssette F., Pépin G. Compared interest between hair analysis and urinalysis in doping control. Results for amphetamines, corticosteroids and anabolic steroids in racing cyclists. J. For. Sci. Int. $1999 ; 107$ : 361-79.

23. Kintz P., Cirimele V., Dumestre-Toulet V., Ludes B. Doping control for nandrolone using hair analysis. J. Pharm. Biomed. Anal. 2001 ; 24 : 1125-30.

24. Thieme D., Grosse J., Sachs S., Mueller R.K. Detection of several anabolic steroids of abuse in human hair. Recent advances in doping analysis. Proceeding of the Manfred Donike Workshop $16^{\text {th }}$ Cologne Workshop on Dope Analysis $15^{\text {th }}$ to $20^{\text {th }}$ March 1998. Sport \& Buch Strauss. $1998 ; 6: 9-29$.

25. Bresson M., Cirimele V., Villain M., Kintz P. Doping control for metandienone using hair analyzed by gas chromatograghy-tandem mass spectrometry. J Chromatogr. B 2006, soumis.

26. Kintz P., Cirimele V., Jeanneau T., Ludes B. Identification of testosterone and testosterone esters in human hair. J. Anal. Toxicol. 1999 ; 23 : 352-6.

27. Cirimele V. Drug concentrations in human hair after bleaching. J. Anal. Toxicol. $1995 ; 19: 331-2$.

28. Kintz P., Cirimele V., Ludes B. Pharmacological criteria that can affect the detection of doping agents in hair. J. For. Sci. Int. 2000 ; $107: 325-34$.

29. Kintz P., Cirimele V, Dumestre-Toulet V., Villain M., Ludes B. Doping control for methenolone using hair analysis by gas chromatography-tandem mass spectrometry. J. Chrom. B. $2002 ; 766$ : 161-7. 